\title{
Prediction of Heat Release Effects on a Mixing Layer
}

Mohammed Farshchi

Sverdrup Technology, Inc.

Lewis Research Center

Cleveland, Ohio

February 1986

Prepared for the Lewis Research Center Under Grant NAS 3-24105

\section{NASก

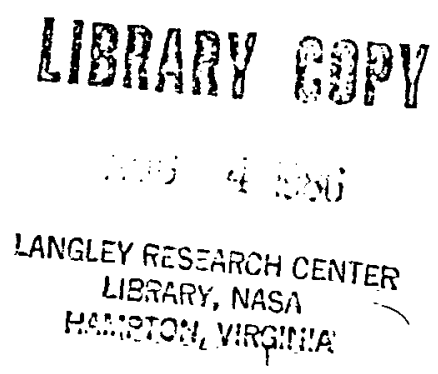


31176013085742 


\title{
PREDICTION OF HEAT RELEASE EFFECTS ON A MIXING LAYER
}

\author{
Mohammed Farshchi \\ Sverdrup Technology, Inc. \\ Lewis Research Center \\ cleveland, Ohio 44135
}

\begin{abstract}
SUMMARY
A fully second-order closure model for turbulent reacting flows is suggested based on Favre statistics. For diffusion flames the local thermodynamic state is related to a single conserved scalar. The properties of pressure fluctuations are analyzed for turbulent flows with fluctuating density. Closure models for pressure correlations are discussed and modeled transport equations for Reynolds stresses, turbulent kinetic energy dissipation, densityvelocity correlations, scalar moments and dissipation are presented and solved, together with the mean equations for momentum and mixture fraction. Solutions of these equations are compared with the experimental data for high heat release free mixing layers of fluorine and hydrogen in a nitrogen diluent.
\end{abstract}

\section{INTRODUCTION}

A test case for the evaluation of a fully second order closure model of turbulent reacting flows with moderate to large heat release has been provided by the recent experiments of Hermanson et a1. (ref. 1) and Mungal and Dimotakis (ref. 2). The dynamic field consists of a two-dimensional mixing layer with gas phase free streams carrying hydrogen in a nitrogen diluent in one stream and fluorine in a nitrogen diluent in the other. The reaction is

$$
\mathrm{H}_{2}+\mathrm{F}_{2} \longrightarrow 2 \mathrm{HF}
$$

and is highly exothermic. The experimental results with the highest heat release correspond to fluorine concentration of up to 6 percent and hydrogen concentration of up to 24 percent, with a maximum adiabatic flame temperature rise of $940 \mathrm{~K}$.

An attempt will be made to assess the possible improvement in the prediction of the above flow fleld by accounting for both velocity and scalar fluctuation time scales and by inclusion of the chemical heat release effects in the modeling of the Reynold stresses, scalar fluxes, and dissipation equations.

\section{Closure Model}

The closure model consists of three parts:

(1) The thermo-chemical model based on the shifting equilibrium flame model (ref. 3). This model requires a fast rate of chemical reaction and relates the value of all thermodynanic variables to a conserved scalar. There are several scalar variables which are conserved in a chemical reaction and can be used as a basis for describing the mixing in a nonpremixed reacting flow. The mass fraction of a given element is such a variable. A normalized 
conserved scalar is referred to as the mixture fraction. Hence the density $\rho$, temperature $T$, and composition $y_{k}$ are local functions of the mixture fraction $f$.

(2) The turbulence or moment model consisting of a set of closed favre averaged equations of order one and two, including the equations for all Reynolds stress components, scalar fluxes, dissipation rates of turbulence $k$ inetic energy, and the mixture fraction variance.

(3) The coupling model which relates the thermo-chemical model and the turbulence model through the probability density function, p.d.f., of the mixture fraction. In general, the form of p.d.f. Will depend on the flow conditions and will be coupled with the chemical heat release. If constraints are imposed by solving the transport equations of the mean and the variance of the mixture fraction, the errors that can be made using an arbitrary form of the p.d.f. are limited. A Favre Beta function will be used here to represent the p.d.f. of the mixture fraction. The means and higher moments of the thermodynamic variables at any point may be obtained from their local relationship with the mixture fraction obtained from the thermo-chemical model and the p.d.f. of the mixture fraction.

NOMENCLATURE

0

$f$

fs

$g_{1}$

k

p

$\tilde{\mathbf{p}}$

R

$\mathrm{T}$

$T_{a d b}$

$T_{\text {amb }}$

u

$\mathrm{U}_{1}$

v

$\underline{x}$

$Y_{k}$

velocity divergence

mixture fraction $f=\tilde{f}+f "$

Stoichiometric value of the mixture fraction

gravitational acceleration vector

turbulent kinetic energy

pressure, $p=\bar{p}+p^{\prime}$

Favre probability density function

ratio of turbulence velocity to scalar time scales

absolute temperature

adiabatic flame temperature

ambient temperature

longitudinal velocity component

velocity vector, $u_{i}=\widetilde{u}_{i}+u_{i}^{\prime \prime}$

transverse velocity component

position vector

mass fraction of the $k^{\text {th }}$ species in the mixture 


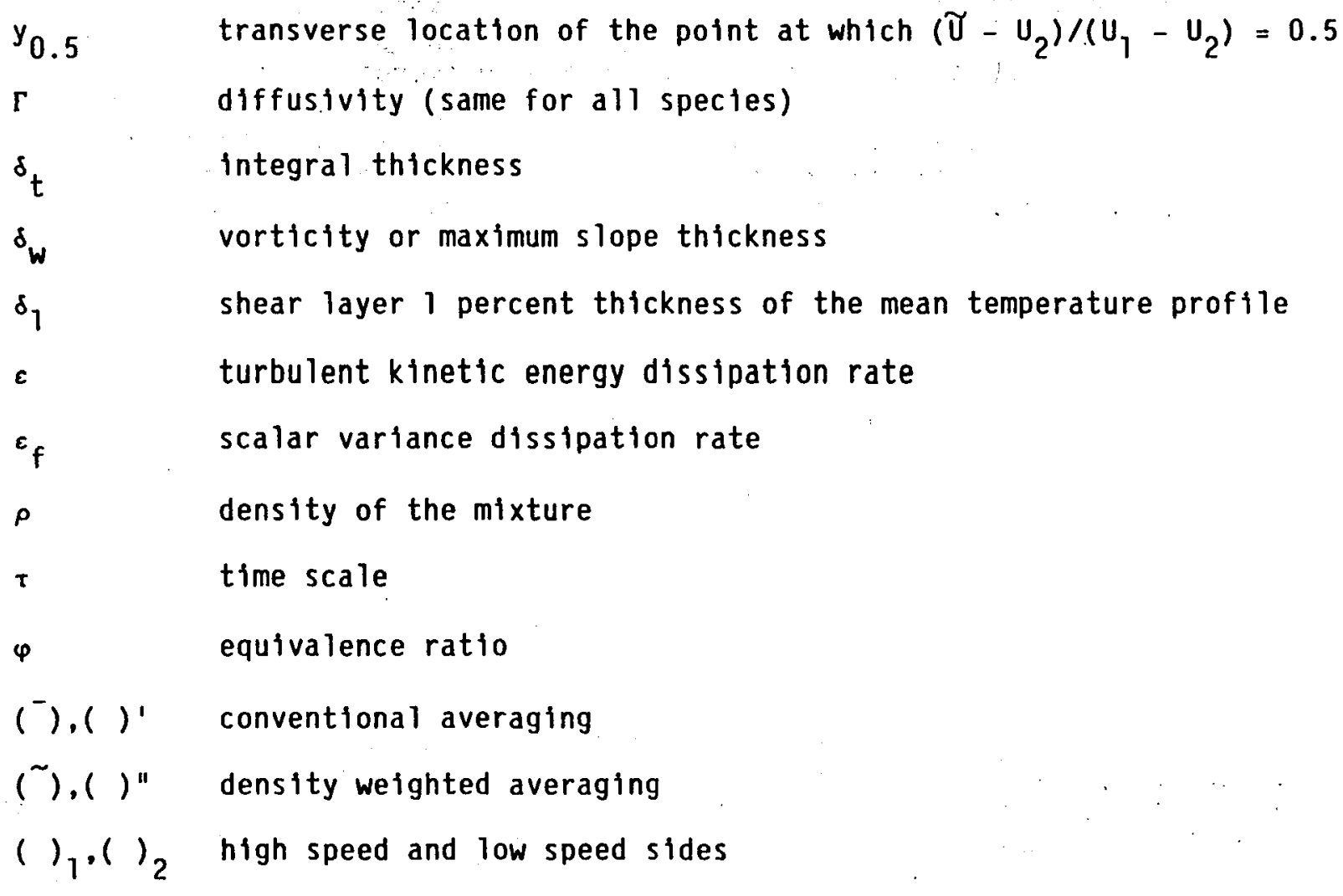

TURBULENCE MODEL

Second-order closure methods are belleved to be the optimum level of closure and have been successfully applied to constant density flows, including buoyancy effects (refs. 4 to 6 ). The presence of the density fluctuations in the variable density or combusting flows results in far more complex equations for the second moments. Application of density weighted (Favre) averaging to variable density flows results in a set of equations which are simflar to those of constant density flows. This extends the use of the well developed constant density second-order closure models to the variable density case and makes available well tested model expressions for many terms in the moment equations (ref. 7). This is, however, not sufficient for the complete closure of the density weighted moment equations, since new correlations involving density and divergence of velocity appear in the set. The exact equations show that the variation of density is felt via three mechanisms: (1) the variation of the mean density and the correlation of density with velocity; (2) the nonzero divergence of velocity; and (3) the correlations involving pressure which are related to density via mass, energy, and state equations. These correlations appear in the transport equations of the Reynolds stresses and scalar fluxes and play an important physical role.

\section{Pressure Equation}

The instantaneous pressure equation follows from mass and momentum balance as

$$
\Delta p=\partial_{t t^{\rho}}^{2}-\partial_{i j}^{2}\left(\rho U_{i} U_{j}\right)+g_{i} \partial_{i} \rho+\partial_{i j}^{2}{ }_{i j}
$$


where $T$ if denotes the Newtonian stress tensor. In contrast to the constant density case, the type of this equation for known velocity is hyperbolic. It describes the change of pressure due to hydromechanical motion and acoustic propagation of waves. The density term however contains not only acoustic variations of pressure but contributions which are due to mixing and chemical reaction. Expanding the first and second terms of above equation and utilizing the instantaneous mass and momentum equations, the above equation can be rewritten as,

$$
\Delta p=-\rho \partial_{f} U_{j} \partial_{j} U_{j}+\frac{1}{\rho} \partial_{j} \rho \partial_{j} p-\rho D_{t} D-\frac{1}{\rho} \partial_{j} \rho \partial_{i} \tau_{i j}+\partial_{i j}^{2} \tau_{i j}
$$

where the substantial derivative is

$$
D_{t}=\partial_{t}+U_{i} \partial_{i}
$$

and

$$
D=\partial_{1} U_{1}=-\frac{1}{\rho} D_{t} \rho
$$

The last two terms in equation (2) represent the molecular viscous effects on pressure and are ignored in comparison to other terms in high Reynolds number flows. The second term in equation (2) results in an integral equation of the Fredholem type of second-order. It can be shown that the iterated kernals for this equation exist, thus a solution can be obtained. This solution is rather complicated, but it produces an integral form with a kernal modified by the density fluctuations. This term will have negligible affect at high Reynolds numbers due to the lack of correlation. The first term on the right-hand side of equation (2) is the well known hydrodynamic source of pressure change, see Chou (ref. 8). This would be the only term appearing in the case of constant density flows. In the context of the thermochemical model for diffusion flames introduced above, density is a local function of the mixture fraction. It is however advantageous to consider $\rho^{-1}$, specific volume, instead of $\rho$. Therefore

$$
D=\rho d_{f} \rho D_{t} f
$$

The pressure equation can be recast in terms of mixture fraction,

$$
\Delta p \approx-\rho \partial_{i} U_{j} \partial_{j} U_{i}-\rho D_{t}\left(\rho d_{f} \rho-D_{t} f\right)
$$

In pure mixing of two gases of different molecular weights the variation of the specific volume as a function of the mixture fraction is close to 1inear, with its second derivative equal to zero. However in diffusion flames this relation is strongly nonlinear near the stoichiometric value of the mixture fraction. To the left and right of this value $\rho^{-1}(f)$ is nearly linear with different slopes. If this local relation is simplified to partially linear with a jump in the slope at the stoichiometric value of the mixture fraction, then the terms involving the first derivative of the specific volume reflect the effect of mixing, with 


$$
d_{f} p^{-1}=a\left(1-H\left(f-f_{s}\right)\right)
$$

and the terms involving the second derivative of the specific volume reflect the effect of chemical heat release, with

$$
d_{f f}^{2} p^{-1}=-a \delta\left(f-f_{s}\right)
$$

where $H\left(f-f_{S}\right)$ denotes the Heaviside function, $\delta\left(f-f_{S}\right)$ the Dirac function as generalized derivative of $H(f)$ and $f_{s}$ is the stoichiometric value of the mixture fraction. Utilizing the specific volume, the equation of conservation of mixture fraction and considering the limiting case of high Reynolds number and Kolmogorov's hypothesis of local isotropy (refs. 9 and 10) the pressure equation for diffusion flames is then reduced to

$$
\Delta p \approx-\rho \partial_{i} U_{j} \partial_{j} \partial_{j} U_{j}+2 \rho^{2} E^{2} d_{f f}^{2}(\ln \rho) d_{f f}^{2} \rho^{-1}
$$

where $E=r \partial_{f} f \partial_{f} f$. Based on the above model and equation (5) the second term on the right hand side of equation (6) is recognized as the heat release contribution to the pressure equation. This term behaves like a Dirac delta function and its magnitude is determined by the location and movement of the instantaneous flame front, $f(\underline{x}, t)=f_{s}$, since only near the flame front it has a large contribution and is small everywhere else. This simplified pressure equation anaiys is indicates that the fluctuations of the pressure contain the contributions of mixing and reaction in addition to velocity fluctuations. Janicka and Lumley (ref. 11) have argued that the mixing part can be neglected at high Reynolds numbers in pure mixing. An order of magnitude analysis shows that in the case of chemical heat release the mixing contribution can also be neglected.

Solution of the Poisson equation, (eq. (6)), for pressure can be obtained by application of Green's theorem. Thus far from walls, where the surface integral may be neglected

$$
P(\underline{x})=P_{1}(\underline{x})+P_{2}(\underline{x})
$$

where

$$
P_{1}(\underline{x})=\frac{1}{4 \pi} \iiint \rho \partial_{j}^{\prime} U_{1} \partial_{i}^{\prime} U_{j} \frac{1}{\left|\underline{x}-\underline{x}^{\prime}\right|} d x_{1}^{\prime} d x_{2}^{\prime} d x_{3}^{\prime}
$$

and

$$
P_{2}(\underline{x})=-\frac{1}{2 \pi} \iiint \rho^{2}\left(r \partial_{1}^{\prime} f \partial_{1}^{\prime} f\right)^{2} d_{f f}^{2}(\ln \rho) d_{f f}^{2} \rho^{-1} \frac{1}{\left|\underline{x}-\underline{x}^{\prime}\right|} d x_{1}^{\prime} d x_{2}^{\prime} d x_{3}^{\prime}
$$

\section{Second-Order Closure}

The complete set of equations constituting a closed system of Favreaveraged moments including all density variation effects will be discussed briefly. 
The mean velocity, at high Reynolds number, satisfies

$$
\partial_{j}\left(\bar{\rho} \widetilde{U}_{i} \tilde{U}_{j}\right)=-\partial_{j} \bar{\rho}-\partial_{j}\left(\overline{\rho u_{j}} \bar{u} u_{j}^{\prime \prime}\right)+\overline{\rho g}_{j}
$$

No closure assumption is required at this level of closure.

The modeled Reynolds stress equation is given as

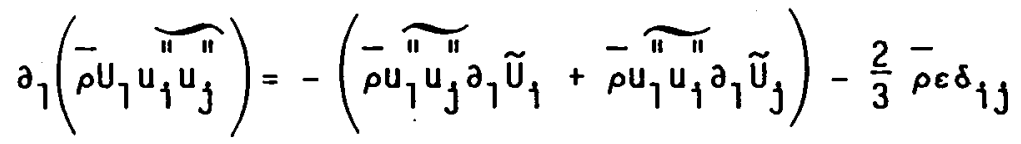

$$
\begin{aligned}
& +\partial_{1}\left(c_{s} \bar{\rho} \frac{k}{\varepsilon} u_{1}^{\prime \prime} u_{m}^{\prime \prime} \partial_{m}\left(\overline{u_{i} u_{j}^{\prime \prime}}\right)\right)+\frac{1}{\rho}\left\{\overline{\rho^{\prime} u_{j}^{\prime \prime} \partial_{j} \bar{p}}+\overline{\rho^{\prime} u_{j}^{\prime \prime} \partial_{j} \bar{p}}\right\}+Q_{i j}^{1}+Q_{i j}^{2}
\end{aligned}
$$

The Kolmogorov assumption of local isotropy at high Reynolds numbers is used here, so that the dissipation tensor is isotropic with its trace equal to twice the turbulence kinetic energy dissipation, $\varepsilon$. The diffusion model of Daly and Harlow (ref. 12) is chosen over the more complicated model suggested by Hanjalic and Launder (ref. 13) due to their equivalent performances in constant density flows. Consideration of the exact solution of the pressure equation given by equations (7) and ( 8 ), suggests the following decomposition of the velocity-pressure gradient correlation

$$
\left.\left.-\overline{\left(u_{i}^{\prime \prime} \partial_{j} p^{\prime}\right.}+\overline{u_{j}^{\prime \prime} \partial_{i} p^{\prime}}\right)=-\overline{\left(u_{i}^{\prime \prime} \partial_{j} p_{1}^{\prime}\right.}+\overline{u_{j}^{\prime \prime} \partial_{i} p_{1}^{\prime}}\right) \overline{\left(u_{i}^{\prime \prime} \partial_{j} p_{2}^{\prime}\right.}+\overline{\left.u_{j}^{\prime \prime} \partial_{i} p_{2}^{\prime}\right)}
$$

The first term on the right-hand side of the above equation is denoted by Q $\}_{j}$ and represents the correlation of the velocity fluctuations with the hydrodynamics part of the pressure fluctuation gradient. This would be the only term present for the incompressible flow case and contains the pure turbulence interaction "return to isotropy," and mean strain rate interaction "fast response" contributions. The closure model of Launder, Reece, and Rodi (ref. 4) is used for this term. In density weighted form this term is given as

$$
\begin{aligned}
& Q_{i j}^{1}=-c_{1} \bar{\rho} \frac{\varepsilon}{k}\left(u_{i j}^{\pi \prime \prime}-\frac{2}{3} \delta_{i j k}\right)-\frac{c_{2}+8}{11}\left(\pi_{i j}-\frac{2}{3} \delta_{i j} \pi\right) \\
& -\frac{8 C_{2}-2}{11}\left(\Delta_{i j}-\frac{2}{3} \delta_{i j} \pi\right)-\frac{30 C_{2}-2}{55} \overline{\rho k}\left(\partial_{j} \tilde{U}_{i}+\partial_{i} \tilde{U}_{j}\right) \\
& -\bar{\rho}_{1} \tilde{u}_{1}\left\{\frac{6 C_{2}+4}{11} \tilde{u}_{i} u_{j}-\frac{40 C_{2}+12}{55} \delta_{1 j} k\right\}
\end{aligned}
$$

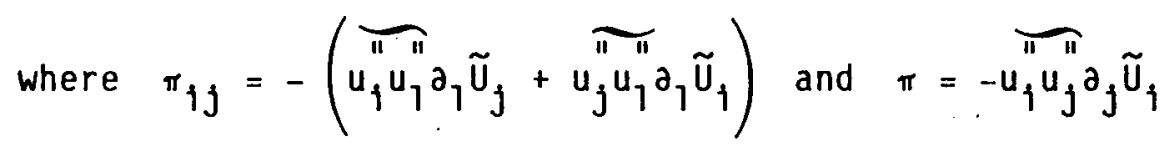

$\Delta_{i j}=-\left(u_{i} u_{i} \partial_{j} \tilde{u}_{1}+\widetilde{u}_{j} u_{1} \partial_{i} \tilde{u}_{1}\right)$ and $k=1 / 2 u_{i}^{\| "} u_{i}$. 
The term Q?f represents the heat release part of the pressurestrain rate correlation. Insight into modeling of this term can be gained by careful analysis of the pressure equation's solution (eq. (8)). Considering just the heat release portion of the pressure solution we have

$$
\begin{aligned}
\overline{-u_{1}^{\prime \prime} \partial_{j} p_{2}}=\frac{1}{2 \pi} \iiint \sqrt{\left(\rho \Gamma \partial_{1}^{\prime} f \partial_{f}^{\prime} f^{2} d_{f f}^{2}(\ln \rho) d_{f f^{\rho}}^{2}\right) u_{1}^{\prime \prime}(\underline{x})} & x \partial_{j}\left(\frac{1}{\left|\underline{x}-\underline{x}^{\prime}\right|}\right) d x_{1}^{\prime} d x_{2}^{\prime} d x_{3}^{\prime}
\end{aligned}
$$

Due to the Dirac delta function behavior of the second derivative of the specific volume near the stoichiometric value of the mixture fraction, this correlation is determined by the location and movement of the instantaneous flame front, $f(\underline{x}, t)=f_{s}$, i.e., only near the flame front does the second derivative of the specific volume contribute to the pressure equation. Therefore, the integral representation of the heat release contribution indicates dependence on the probability of the flame sheet being present at a given location. On the other hand, the probability of mixture fraction being in an interval about the stoichiometric value, $\widetilde{\underline{P}}\left(f_{s} ; \underline{x}\right) d f$, is zero for pure oxidizer, where $f=0$, and grows quickly with fuel concentration. It reaches its maximum at the flame front region and declines to zero in the pure fuel region, where $f=1$. This suggests that $\partial_{1} \widetilde{\widetilde{P}}\left(f_{s} ; \underline{x}\right)$ is large and positive on the entrainment side of the flame and becomes small and negative past the flame front. Considering the dimensional properties of equation (12) it becomes clear that there are two time scales affecting this term. One is the scalar fluctuation time scale defined by the scalar variance and its dissipation, the other one is the dynamic or velocity fluctuation time scale, which is related to the turbulence large eddy structures. If tensorial properties and consistency with the limits of zero fluctuations and pure mixing without reaction are required, the following possible closure model emerges:

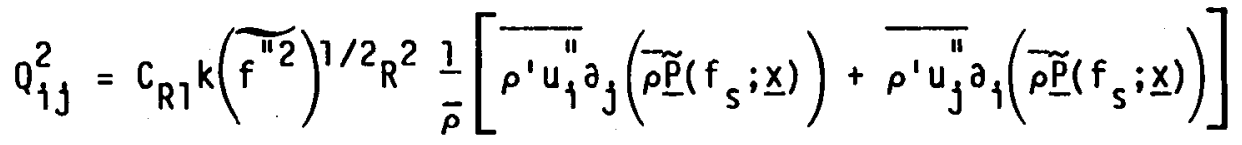

where $R=2 \varepsilon_{f} / f^{\pi / 2} \mathrm{k} / \varepsilon$ denotes the ratio of turbulence time scale to scaler fluctuations time scale and $\varepsilon_{f}=\Gamma \partial_{j} f^{\prime \prime} \partial_{j} f "$ is the rate of dissipation of the scalar variance. Beguler, Dekeyser, and Launder (ref. 14) showed from a survey of several nonisothermal, nonreacting turbulent shear flows that the time scale ratio was approximately equal to 2.0 . The implied constancy of $R$ is used in most work aimed at calculating scalar variances. However based on the evidence to the contrary provided by Warhaft and Lumley (ref. 15) an exact transport equation for the scalar dissipation is derived and modeled, so that $R$ can be determined at each point.

The constant $C_{R 1}$ can be estimated as

$$
C_{R 1} \sim c * \frac{1}{8 \pi} d_{f f}^{2} \ln \rho
$$

where $C^{\star}$ is a measure of correlation among the terms in the integral (eq. (12)) and should have a value much less than one due to the degree of 
statistical independence implied in the modeling of this term. off in $\rho$ is a chemically dependent term that varles from zero (for nonreacting cases) to the order of 100 for reactions with large heat release. Therefore the value of $C_{R 1}$ depends on the energy release in the flow fleld. For the hydrogen - air flame of Dibble, Kollmann, and Schefer (ref. 16) a value of 1.25 is recommended (ref. 10).

The rate of turbulence kinetic energy dissipation determines the dynamic length and time scales and satisfies the following modeled equation

$$
\begin{aligned}
& \partial_{j}\left(\bar{\rho} \tilde{U}_{i} \varepsilon\right)=\partial_{j}\left(c_{\varepsilon} \bar{\rho} \frac{k}{\varepsilon} u_{i}^{\prime \prime} u_{j}^{\prime \prime} \partial_{i} \varepsilon\right)-c_{\varepsilon 1} \bar{\rho} \frac{\varepsilon}{k} u_{i}^{\prime \prime} u_{j}^{\prime \prime} \partial_{j} \tilde{U}_{i}-c_{\varepsilon 2} \bar{\rho} \frac{\varepsilon}{k}-\frac{2}{3} \bar{\rho} \varepsilon \partial_{i} \tilde{U}_{i} \\
& +C_{\varepsilon 3} \frac{\varepsilon}{k}\left(\frac{\overline{\rho u_{i}^{\prime \prime}}}{\bar{\rho}}\right) \partial_{1} \bar{p}+C_{R 2}\left(\overline{f^{\prime \prime 2}}\right)^{1 / 2} R^{2} \frac{\overline{\rho u_{i}^{\prime \prime}}}{\bar{\rho}} \partial_{j}\left(\overline{\rho P}\left(f_{s} ; \underline{x}\right)\right)
\end{aligned}
$$

where the last term contains the effect of chemical heat release on dissipation. This complex process is tentatively taken into account by analogy with the corresponding term (eq. (13)) for the normal stresses.

Density - Velocity correlations appear both directly and through the modeled terms in the Reynolds stress transport equation (10). Derivation of exact equations and the closure of the equations are discussed in detail by Farshchi (ref. 10). The modeled transport equations for these correlations is given by

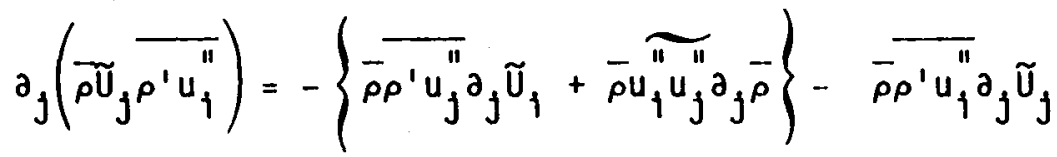

$$
\begin{aligned}
& +\partial_{j}\left(c_{s} \tau \rho u_{j} u_{1} \partial_{1} \rho^{\prime} u_{i}^{\prime \prime}\right)-2 c_{s} \tau u_{j}^{\prime \prime} u_{1} \partial_{1}\left(\overline{\rho^{\prime} u_{i}^{\prime \prime}}\right) \partial_{j} \bar{\rho}+\frac{\overline{\rho^{\prime 2}}}{\bar{\rho}} \partial_{j} \bar{p}
\end{aligned}
$$

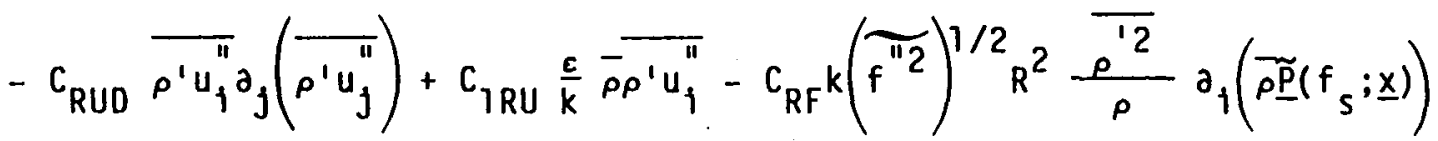

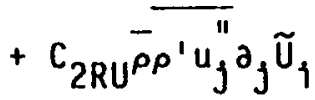

$\tau$ is the turbulent time scale, which is traditionally set equal to the turbulence time scale $k / \varepsilon$. However it can also be the time scale of scalar fluctuations or a combination of the two time scales (ref. 17). $\rho^{12}$ is the variance of density and is obtained directly using the thermo-chemical model and the appropriate weighted integration of the mixture fraction p.d.f.

The transport equation of the mixture fraction's mean value requires no modeling and is given as 


$$
\partial_{i}\left(\widetilde{\rho U}_{i} \tilde{f}\right)=-\partial_{i}\left(\overline{\rho u}_{i}^{\prime \prime}\right)
$$

where the molecular diffusion effects are neglected in comparison with the turbulence diffusion at high Reynolds numbers.

Next a transport equation for the scalar flux vector, u"f", is derived and modeled (ref. 6). The scalar pressure gradient terms (pressure scrambling) are modeled using the same line of argument used for velocity-pressure gradient correlations in the Reynolds stress equation. The modeled form of this equation is given as

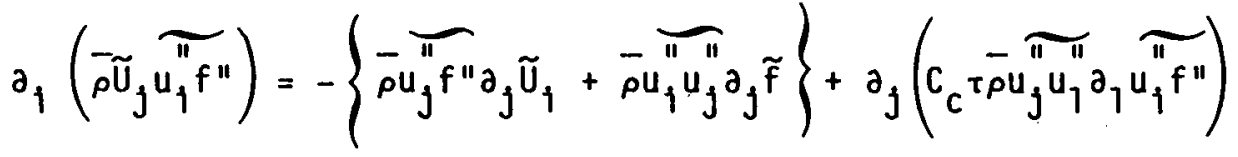

$$
\begin{aligned}
& +\frac{1}{\rho} \bar{\rho} \bar{f} " \partial_{i} \bar{p}-c_{1 c} \frac{1}{\tau} \overline{\rho u_{i}^{\prime \prime} f \prime}+c_{2 c} \overline{\rho u_{j}^{\prime \prime} f " \partial_{j}} \tilde{u}_{i} \\
& +C_{R F} k\left(\widetilde{f^{\prime \prime}}\right)^{1 / 2} R^{2} \frac{\overline{\rho^{\prime} f^{\prime \prime}}}{\bar{\rho}} \partial_{i}\left(\bar{\rho} \underline{\tilde{P}}\left(f_{s} ; \underline{x}\right)\right)
\end{aligned}
$$

$\overline{\rho^{\prime} f \prime}$ appearing in the above equation can be obtained from the p.d.f. of the mixture fraction.

Mixture fraction variance appears in the Beta function representing the probability density of the mixture fraction. For high Reynolds number flows the modeled mixture fraction variance equation is expressed as

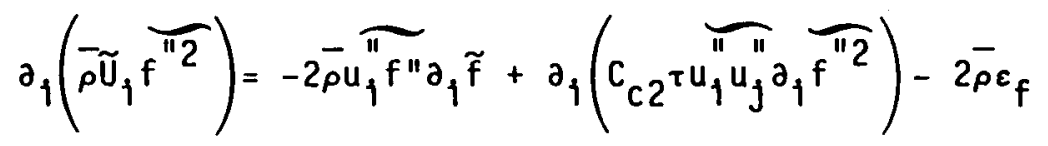

The variance of the mixture fraction is dissipated by molecular action at small scales. This dissipation rate is also the key to determination of scalar fluctuation time scale. The exact form of the scalar dissipation equation and its modeling is discussed in detalls by Farshchi et al. (ref. 18). However, regrouping some of the exact terms and applying an order of magnitude analysis and ignoring terms of order $R_{t}^{-1}$ and 1ess, where $R_{e}=k^{2} / v \varepsilon$ is the turbulence Reynolds number, Farshchi (ref. 10) shows thatfor high heat release cases there is a source along with a sink of the scalar dissipation. The chemical source term for diffusion flames is given by

$$
\overline{d_{f f}^{2} \rho\left(\Gamma \partial_{q} f " \partial_{q} f "\right)^{2}} \approx c_{p 3} \bar{\rho} \tilde{P}\left(f_{s} ; x\right) \varepsilon_{f}^{2} \frac{1}{f^{\prime \prime 2}}(\widetilde{f " 2})^{1 / 2}
$$

with $c_{p 3}=1.0$. The completely modeled scalar dissipation equation is then given by: 


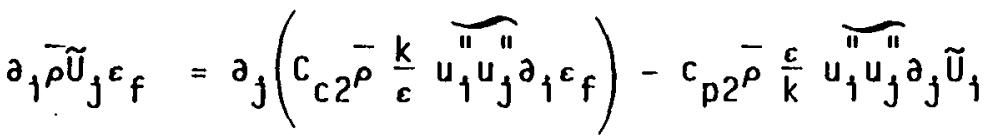

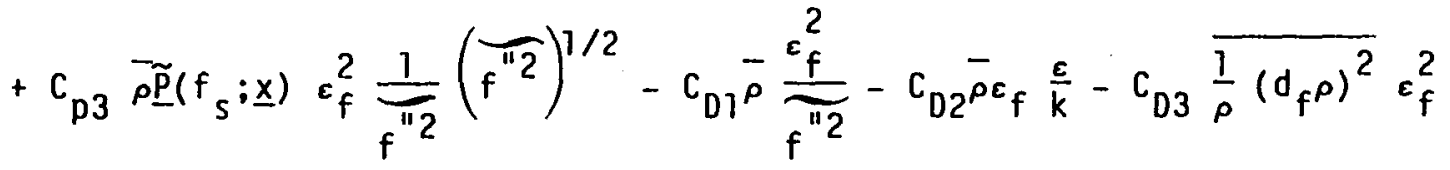

$$
\begin{aligned}
& -C_{p l} \bar{\rho} \frac{\varepsilon_{f}}{f^{\prime \prime 2}} u_{j}^{\prime \prime} f \partial_{j} \tilde{f}
\end{aligned}
$$

A11 constants used in above equations are summarized in table I.

\section{APPLICATION AND COMPARISON WITH MEASUREMENTS}

Hermanson's (ref. 19) experimental studies of the effect of heat release in a planar, gaseous reacting mixing layer formed between free streams containing hydrogen and fluorine in nitrogen diluents are used for comparison. The density ratio of the free streams is kept equal to one by using as diluent a mixture of nitrogen and a small amount of helfum, on the fluorine side, and a mixture of nitrogen with small amount of argon on the hydrogen side. The nominal high speed, hydrogen containing side, flow velocity is $22 \mathrm{~m} / \mathrm{s}$ and a free-stream speed ratio of $U_{2} / U_{1}=0.4$ is maintained. The high speed free-stream turbulence level was measured to be about $2 / 3$ percent. The measuring station was positioned $0.457 \mathrm{~m}$ downstream of the splitter plate tralling edge. The Reynolds number at the measuring station based on the high speed free-stream velocity, the downstream distance and the cold free-stream kinematic viscosity was $\operatorname{Re}_{x}=6 \times 10^{5}$. The Reynolds number based on high speed boundary layer momentum thickness just upstream of the tralling edge was estimated to be about 240 .

Two cases of moderate and high heat release are considered, corresponding to two equivalence ratios (ref. 2):

(1) $\varphi=1$, corresponding to fluorine concentration of 6 percent and hydrogen concentration of 6 percent, with a maximum adiabatic flame temperature of $860 \mathrm{~K}$.

(2) $\varphi=1 / 4$, corresponding to fluorine concentration of 6 percent and hydrogen concentration of 24 percent, with a maximum adiabatic flame temperature of $1240 \mathrm{~K}$. Thermodynamic tables relating density and temperature to the mixture fraction are obtained using CEC83 (ref. 20) computer program.

\section{Numerical Method}

The numerical method used for this calculation is a modified version of the Patankar and Spalding method (ref. 21) where all the first order moments are defined on node points, and all higher order moments are defined at midpoints. The given initial velocity profiles have Blasius form and agree with the boundary layer momentum thickness value reported by the experiment. The initial dissipation rate, $\varepsilon$, and the shear stress, " "v", are determined 
utlilizing the turbulence equilibrium assumption, a simple gradient fiux model with molecular kinematic viscosity, and the assigned velocity profile. Next the well known turbulence viscosity model,

$$
\mu_{t}=0.09-\frac{k^{2}}{\varepsilon}
$$

with laminar viscosity is used to determine initial turbulent kinetic energy profile. The normal stresses are equi-partitioned. The inftial mean mixture fraction has a step profile and all other turbulent correlations are set to zero. The longitudinal mean pressure gradient must be prescribed analytically in terms of the gradient of the outer boundaries velocities and is zero here. The transverse pressure gradient cannot be prescribed and is approximated by a simplified version of the mean transverse momentum equation as

$$
\partial_{y} \bar{p}=-\partial_{y}\left(\bar{\rho} v^{\pi 2}\right)
$$

The upper bound of the marching step of the main procedure is determined at each forward position with respect to the value of a characteristic thickness of the mixing layer. To solve a typical problem with this 13 equation turbulence model over a distance of $0.9 \mathrm{~m}$ wtth 80 node point in the cross direction, the time needed on an IBM 370 is about $30 \mathrm{~min}$ CPU. The p.d.f. integration for the calculation of mean density, temperature, and other mixture fraction moments at each node takes $2 / 3$ of above time.

\section{Discussion of the Results}

The initial prediction of the homogeneous cold flow mixing layer resulted in a mixing layer thickness 35 percent below its experimental value at the axial measuring station. To validate the model constants and the method used here and due to the lack of velocity correlations measurements by Hermanson (ref. 19) It was decided to use Browand and Latigo's (ref. 22) experimental study. Two measures of thickness of the turbulent mixing region are employed. First is the integral thickness defined as

$$
\delta_{t}=\frac{1}{(\Delta U)^{2}} \int_{-\infty}^{\infty}\left[U_{1}-U(y)\right]\left[U(y)-U_{2}\right] d y
$$

Second is the vorticity thickness defined as

$$
\delta_{w}=\frac{(\Delta U)}{\left(\partial_{y} U\right)_{\max }}
$$

Figure 1 shows that there is about 16 percent difference between the experimental and calculated values of the normalized integral thickness. The axial rate of change of vorticity thickness is experimentaliy estimated to be between 0.15 and $0.17 \lambda$, where $\lambda=\left(U_{1}-U_{2}\right) /\left(U_{1}+U_{2}\right)$. The calculated value, $d_{X} \delta_{w}=$ 
$0.148 \lambda$, is at most 13 percent less than the experimental value. Comparison of normalized mean velocity and turbulence shear stress are presented in figures 2,3 , and 4 . They show good agreement with experiment except near the high speed side where the calculation shows a faster approach to the outer values.

Physically the spread of the mixing layer downstream of the splitter plate is determined by two different mechanisms. The spreading rate of the mixing layer at dynamical equilibrium stage is one mechanism. This is reasonably well predicted by the calculation. The other and more crucial mechanism, which is not predicted by the calculation, involves the transition from attached boundary layers on the splitter plate to free mixing layer developing downstream of it. If the boundary layers are laminar then there is also a transition from laminar to turbulent flow in the developing region of the mixing layer. Birch (ref. 23) has attempted to model the developing region of the mixing layer by a multi-length scale gradient flux turbulence model. However, present calculations indicate that a single-length scale second-order model along with substantially reduced value of the initial turbulent kinetic energy dissipation would result in an increase of the spreading in the initial developing region. Even though the final spreading rate in the similarity region is the same as before, the thickness of the mixing layer is larger by up to 25 percent. The major short coming of this treatment is that the amount of initial reduction of $\varepsilon$ is not the same for all flow conditions and is a function of velocity ratio and character of the boundary layers on the splitter plate. Since in the study of heat release effects only relative values of the spreading are of importance the basic model without any modification can be used.

To examine the contribution of heat release terms introduced by pressure fluctuation correlations and those appearing in the scalar dissipation equation two sets of model constants are examined:

(I) Constants $C_{R 1}, C_{R 2}, C_{R F}, C_{D 3}$, and $C_{p 3}$ are all set to zero

(II) The values given in table I are used for above constants

The rest of the constants are kept the same in both cases.

The comparison of mean axial velocities (fig. 5), and turbulent shear stresses (fig. 6), obtained for the large heat release case, $\varphi=1 / 4$, utilizing models (I) and (II) indicate that there is a little difference between the two models and the improvement obtained by the application of model (II) is almost negligible. This means that the heat release portion of the pressure fluctuation correlations has little effect on the turbulent velocity field. This result can also be shown by the fact that at each level of heat release, models (I) and (II) predict very close values for the spreading rate of the mixing layer. The spreading rate, however, decreases with the heat release. Spreading rate is defined by $d\left(y_{0.1}-y_{0.9}\right) / d x$, 1.e., the rate of spread of the distance between the points at which $U$ equals $0.1\left(U_{1}-U_{2}\right)+U_{2}$ and $0.9\left(U_{1}-U_{2}\right)+U_{2}$. Table II summarizes the spreading rates at different levels of heat release.

The moderate heat release case causes about 10 percent reduction in the spreading rate, however the increase in the heat release has only produced about 3 percent further reduction. The spreading rate levels off as the heat release is increased. This leveling corresponds with the leveling of the mean 
density reduction reported by Hermanson (ref. 19) and leads to the conclusion that probably the single most important cause of the spreading rate reduction with heat release is the reduction of density and the subsequent reduction of the turbulent shear stress, $-\bar{p} u^{\prime \prime} v^{\prime \prime}$. Turbulent shear stresses normalized by free-stream density and velocity difference are compared in figure 7 . There is a considerable reduction of shear stress between the cold and moderate heat release cases. Further increase of the heat release, however, results in a small reduction in the value of shear stress.

Hermanson (ref. 19) reported a monotonic decrease of the vorticity thickness, $\delta_{w}$, with heat release without any influence of the equivalence ratio on this trend. The present equilibrium flame formulation of the chemical reaction is however dependent on the stoichtometric value of the mixture fraction and therefore on the equivalence ratio, $\varphi$. For 6 percent fluorine and $\varphi=1$, the stoichiometric value of the mixture fraction is about 0.5 and for $\varphi=1 / 4$ it is about 0.2 . It is in the neighborhood of this value that the mixture density reaches its minimum value. Figures 8 and 9 show the differences in the density variance distributions and the mean density minimum value location for the two cases of $\varphi=1$ and 1/4. The movement of the mean density minimum value causes the shift in the profile of turbuient shear stress and the location of its peak value as displayed in figure 7 . Since the velocity profile is dependent on the gradient of the turbulent shear stress and not its absolute magnitude, the vorticity thickness predicted for the case of moderate heat release, $\varphi=1$, is smaller than the value predicted for the high heat release case, $\varphi=1 / 4$. Hermanson's (ref. 14) experimental results for 6 percent fluorene and $\varphi=1$ also show some scatter in vorticity thickness measurements with some values that are smaller than those measured for the higher heat release case, $\varphi=1 / 4$. These values are compared with calculated results in table III.

Considering the general trend of the experimental vorticity thickness behavior and assuming that above scatter is within acceptable measurement accuracy bounds, one concludes that the above discrepancy between the predicted and measured resuits and the dependence on the stofchiometric value of the mixture fraction can be resolved by accounting for intermittency effects by such methods used by Kent and Bilger (ref. 24). Byggstoyl and Kollmann (ref. 25) have presented a model for the prediction of intermittency and initial exploratory calculations with such models point to better predictions.

Figure 10 compares the predictions of model (I) and (II) for the ratio of scalar to velocity field time scales, indicating that the influence of modifications included in model (II) is larger on the scalar field than the velocity field. Even though the difference in density predictions (fig. 11), is not large, the density variance and the mixture fraction variance predictions (figs. 12 and 13), show as much as 36 percent jump in their values with mode 1 (II) near the stoichlometric value of the mixture fraction. This change in the magnitude of the mixture fraction variance causes more than 10 percent reduction in the peak value of the predicted temperature which corresponds very we 11 with the experimental measurement, as shown in figure 14. The transverse coordinate in this figure is normalized by shear layer 1 percent thickness, which is defined as the transverse width of the layer at which the mean temperature is 1 percent of the maximum mean temperature rise. The shift between the predicted and measured temperature proftles is symmetric on both sides and is expected to be a consequence of a fixed horizontal high speed upper side wall and diverging low speed lower side wall in the experiment. 
Comparison with the limited experimental results avallable indicate that the modeling approach used here is a step in the right direction. However, comprehensive measurements of the scalar field quantities, such as mixture fraction and density variances and scalar-velocity correlations, are needed to examine and fully evaluate the contribution of the model presented here.

\section{CONCLUSIONS}

A fully second-order closure model for reacting turbulent flows have been extended to include the effects of heat release on he turbulence flow field. This was done by analyzing the exact pressure equation of a turbulent nonpremixed flame. The scalar field time scale has also been directly calculated by developing an equation for the dissipation of scalar variance which also includes the heat release effects. Two models, (I) not including heat release terms and (II) including the heat release terms were compared and indicate that: (a) The velocity fleld predictions are very similar in both models, correctly predicting the reduction in mixing layer spread rate with heat release. The scalar field results of model (II) are in better agreement with the experimental data yielding a 10 percent improvement in temperature predictions. (b) The time scale ratio is sensitive to density fluctuations. This points to a correct inclusion of heat release effects in the equation of scalar variance dissipation. Nevertheless, it would be premature to draw any conclusion about universality of such a closure and the values of the constants used here. Further experiments are needed to support or to improve present assumptions and modeling.

\section{REFERENCES}

1. Hermanson, J.C., Munga 1, M.G., and Dimotakis, P.E., "Heat Re Tease Effects on Sheat Layer Growth and Entrainment," AIAA Paper 85-0142, Jan. 1985.

2. Mungal, M.G. and Dimotakis, P.E., "Mixing and Combustion With Low Heat Release in a Turbulent Shear Layer," Journal of Fluid Mechanics, Vol. 148, Nov. 1984, pp. 349-382.

3. B1lger, R.W., "Turbulent Flows With Nonpremixed Reactants," Turbulent Reacting Flows, P.A. Libby and F.A. Williams, eds., Springer, pp. 65-113.

4. Launder, B.E., Reece, G.J., and Rodi, W., "Progress in the Development of a Reynolds-Stress Turbulence Disclosure," Journal of Fluid Mechanics, Vo1. 68, Part 3, pp. 537-566.

5. Lumley, J.L., Prediction Methods for Turbulent Flows: Introduction, Von Karman Institute Lecture Series 76, Rhode St. Genese, Belglum, 1978.

6. Launder, B.E., "Heat and Mass Transport," Turbulence, P. Bradshaw, ed., Springer, Ber1in, 1976, pp. 232-287. 
7. Jones, W.P. "Models for Turbulent Flows With Variable Density and Combustion," Prediction Methods for Turbulent Flows, W. Kollman, ed., Hemisphere, Washington, O.C., 1980, pp. 379-421.

8. Chou, P.Y., "On Velocity Correlations and the Solutions of the Equations of Turbulent Fluctuation," Quarterly of Applied Mathematics, Vol. 3, 1945. pp. 38-54.

9. Kollmann, W., "Mass Transport in Turbulent Flames," Paper Ms-7250, AIChE Meeting, Los Angeles, CA, 1982.

10. Farshcht, M., "Second-Order Closure for Turbulent Flames," Ph.D. Thesis, University of California, Davis, 1984.

11. Janicka, J. and Lumley, J.L., "Second-Order Modeling in Non-Constant Density Flows," Report FDA 81-01, Cornell Univ. Jan. 1981.

12. Daly, B.J. and Harlow, F.H., "Transport Equations in Turbulence," Phystcs of Fluids, Vol. 13, No. 11, Nov. 1970, pp. 2634-2649.

13. Hanjalic, K. and Launder, B.E., "A Reynolds Stress Model of Turbulence and its Application to Thin Shear Flows," Journal of Fluid Mechanics, Vol. 52, Part 4, 1972, pp. 609-638.

14. Bequier, C., Dekeyser, I. and Launder, B.E., "Ratio of Scalar and Velocity Dissipation Time Scales in Shear Flow Turbulence," Physics of Fluids. Vol. 21, No. 3, Mar. 1978, pp. 307-310.

15. Warhaft, 2. and Lumley, J.L., "An Experimental Study of the Decay of Temperature Fluctuations in Grid-Generated Turbulence," Journal of Fluid Mechanics, Vol. 88, Part 4, 1978, pp. 659-684.

16. Dibble, R.W., Kollmann, W. and Schefer, R.W., "Conserved Scalar Fluxes Measured in a Turbulent Nonpremixed Flame by Combined Laser Doppler Velocimetry and Laser Raman Scattering," Combustion and Flame, Vol. 55 , No. 3, Mar. pp. 307-321.

17. Elghobashi, S.E. and Launder, B.E. "Turbulent Time Scales and the Dissipation Rate of Temperature Variance in the Thermal Mixing Layer," Physics of Fluids, Vol. 26, No. 9, Sept. 1983, pp. 2415-2419.

18. Farshch1, M., Kollmann, W., Dibble, R.W. and Schefer, R.W., "Second-Order Closure for Turbulent Nonpremixed Flames," Fifth Symposium on Turbulent Shear Flows, Corne11 University, Ithaca, NY, 1985, pp. 10.19-10.25.

19. Hermanson, J.C., "Heat Release Effects in a Turbulent, Reacting Shear Layer," Ph.D. Thesis, California Institute of Technology, 1985.

20. Gordon, S. and McBride, B.J., "Computer Program for Calculation of Complex Chemical Equilibrium Compositions, Rocket Performance, Incident and Reflected Shocks, and Chapman-Jouquet Detonations," NASA SP-273, 1971.

21. Patankar, S.V. and Spalding, D.B., Heat and mass transfer in boundary layers, Intertext, London, 1970. 
22. Browand, F.K. and Latigo, B.0., "Growth of the Two-Dimensional Mixing Layer From a Turbulent: and Nonturbulent Boundary Layer," Physics of

Fluids, Vol. 22, No. 6, June 1979, pp. 1011-1019.

23. Birch, S.F., "Mult1-Length Scale Turbulence Mode1s," AFOSR-84-0249TR, 1983. (AD-A140527)

24. Kent, J.H. and Bilger, R.W., "The Prediction of Turbulent Oiffusion Flame Fields and Nitric Oxide. Formation," 16th Symposium (International) on Combustion. The Combustion Institute, Pittsburgh, PA, 1977, pp. 1643-1656.

25. Byggstoyl, S. and Kollmann, W. "A Closure Model for Coniditioned Stress Equations and its Application to Turbulent Shear Flows, " (submitted to Physics of Fluids) 1986. 
TABLE I. - MODEL CONSTANTS

\begin{tabular}{|ccccccc|}
\hline$C_{s}$ & $C_{1}$ & $C_{2}$ & $C_{R 1}$ & $C_{c}$ & $C_{\varepsilon 1}$ & $C_{c 2}$ \\
0.2 & 1.6 & 0.43 & 1.25 & 0.15 & 1.45 & 1.90 \\
$C_{c 3}$ & $C_{R 2}$ & $C_{1 R U}$ & $C_{2 R U}$ & $C_{R F}$ & $C_{R U D}$ \\
1.0 & 1.25 & 4.0 & 0.5 & 1.25 & 1.0 \\
$C_{C}$ & $C_{1 C}$ & $C_{2 C}$ & $C_{C 2}$ & $C_{D 1}$ & $C_{D 2}$ & $C_{D 3}$ \\
0.18 & 3.2 & 0.5 & 1.18 & 2.2 & 0.8 & 1.0 \\
$C_{p 1}$ & $C_{p 2}$ & $C_{p 3}$ & & & & \\
1.8 & 0.8 & 1.0 & & & & \\
\hline 1
\end{tabular}

TABLE II. - SPREADING RATES OF THE MIXING LAYER AT DIFFERENT LEVELS OF

HEAT RELEASE

\begin{tabular}{|l|c|}
\hline \multicolumn{1}{|c|}{ Heat release level } & $d\left(y_{0.1}-y_{0.9}\right) / d x$ \\
\hline Cold flow & 0.0562 \\
Moderate heat release & .0505 \\
$\left(\varphi=1, T_{a d b}=860 \mathrm{~K}\right)$ & .0488 \\
High heat release & \\
$\left(\varphi=1.4, T_{a d b}=1240 \mathrm{~K}\right)$ & \\
\hline
\end{tabular}

TABLE III. - COMPARISON OF NORMALIZED VORTICITY THICKNESS $\delta_{W} / \delta_{W O}$ WITH MEASUREMENTS

[ $\delta_{w o}$ is the cold flow vorticity thickness.]

\begin{tabular}{|c|c|c|}
\hline & $\begin{array}{c}\text { Experimental (ref. 14) } \\
\text { measurements }\end{array}$ & $\begin{array}{c}\text { Present } \\
\text { calculation }\end{array}$ \\
\hline$\varphi=1$ moderate & 0.7645 & 0.8874 \\
heat release & .8066 & .8814 \\
& .9067 & .8814 \\
$\varphi=1 / 4$ high & .8826 & .9627 \\
heat release & & \\
\hline
\end{tabular}




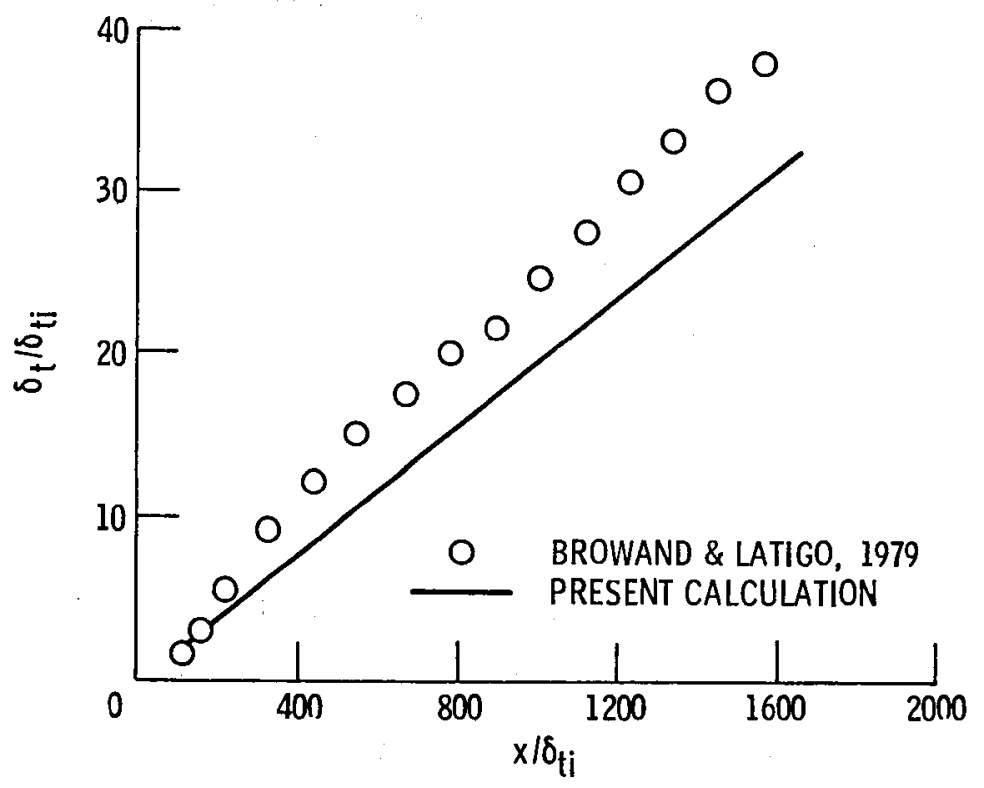

Figure 1. - Normalized local integral thickness as a function of downstream distance for $\lambda=0.695$ and $\delta_{\mathrm{ti}}=0.46 \mathrm{~mm}$.

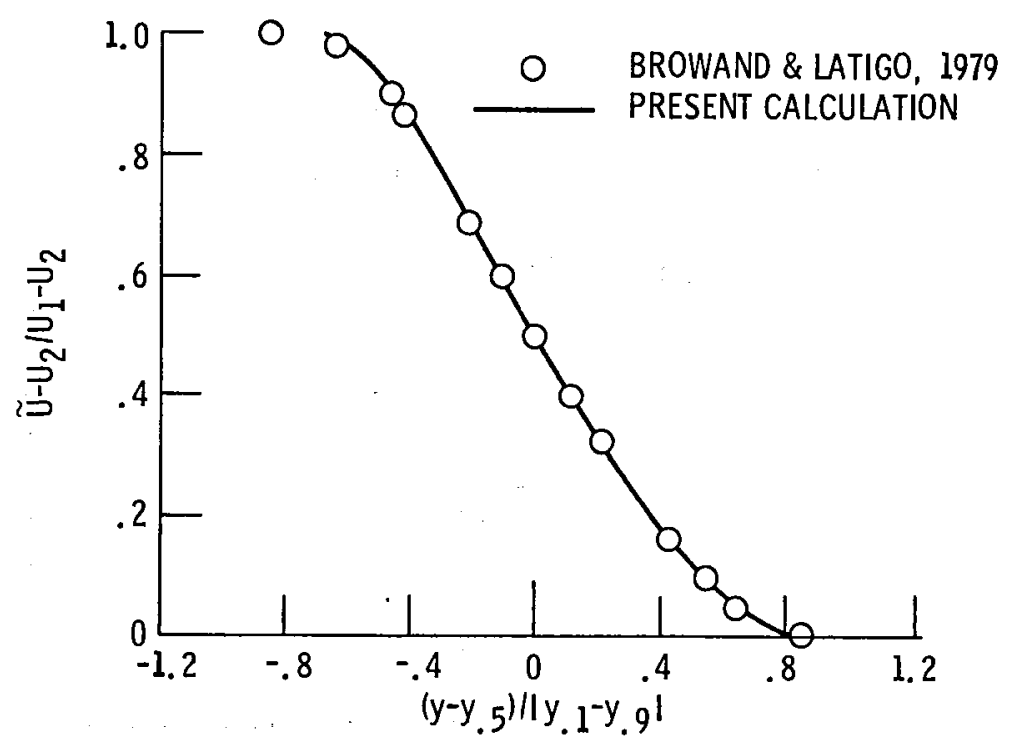

Figure 2. - Longitudinal mean velocity. 


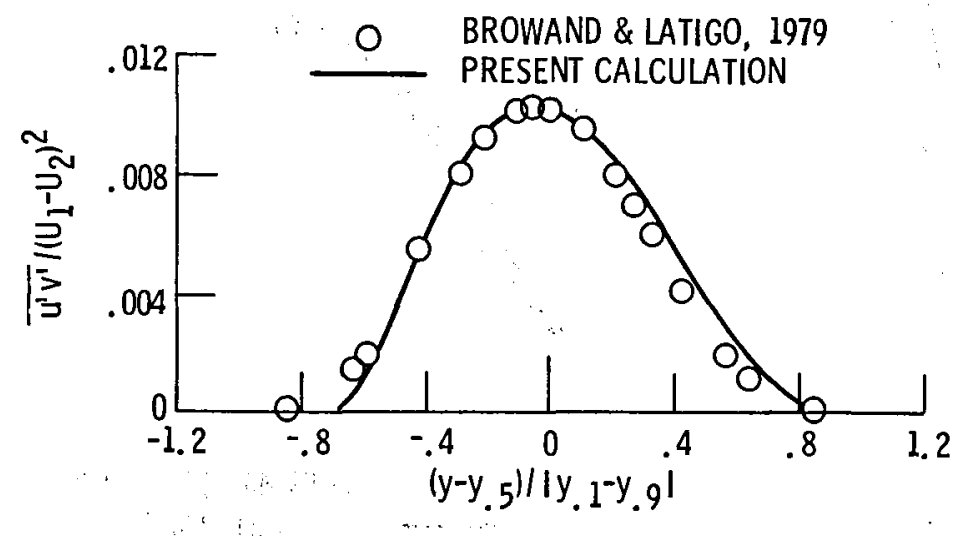

Figure 3. - Distribution of shear stress.

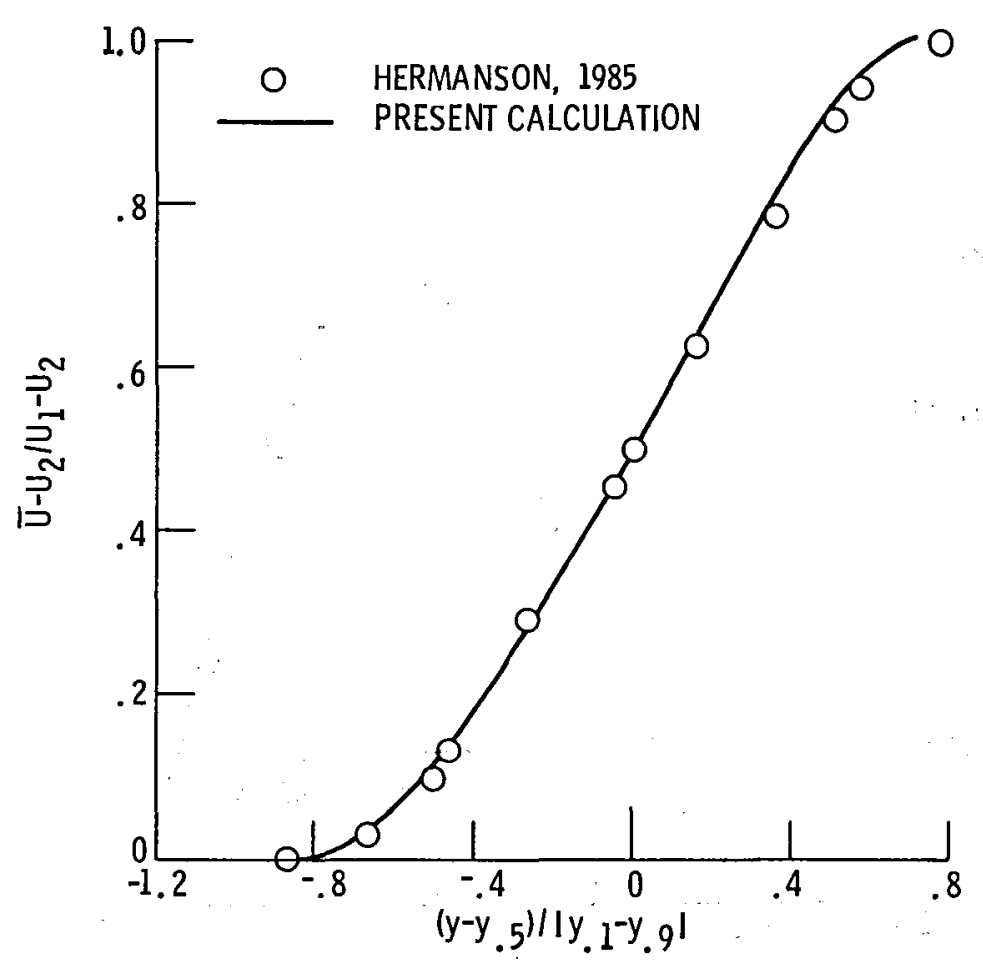

Figure 4. - Longitudinal mean velocity, cold flow. 


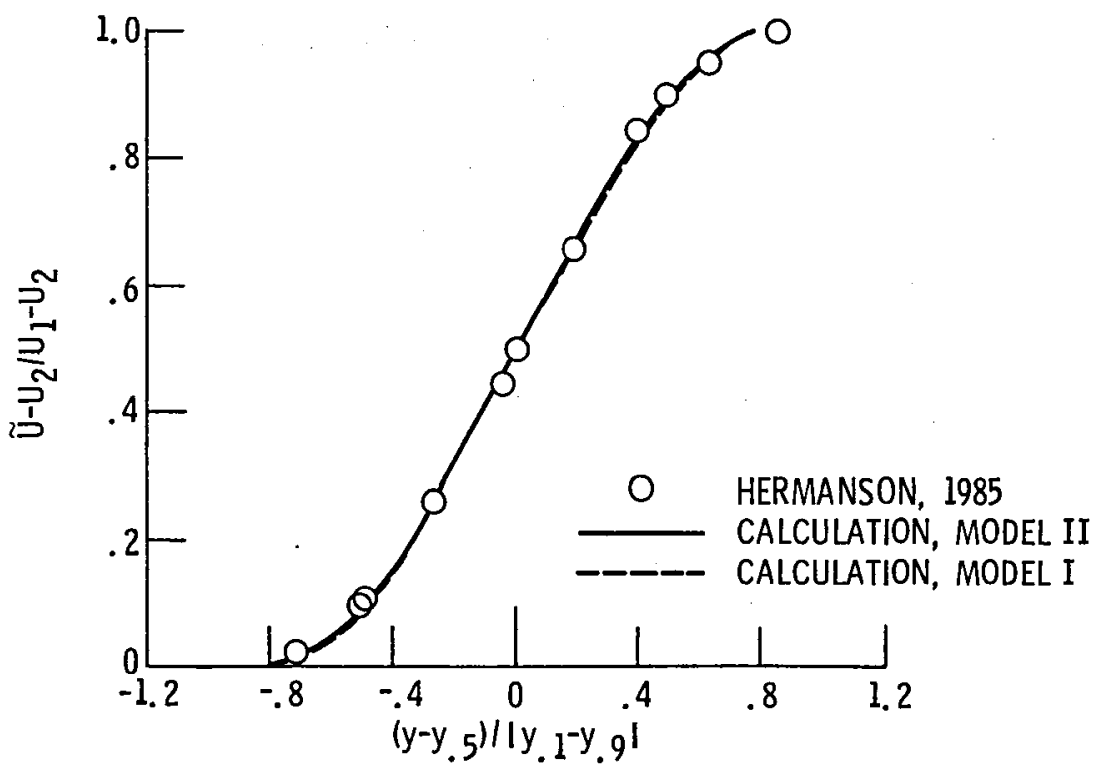

Figure 5. - Comparison of velocity prediction of models I and II with the experiment for the high heat release case, $\varphi=1 / 4$.

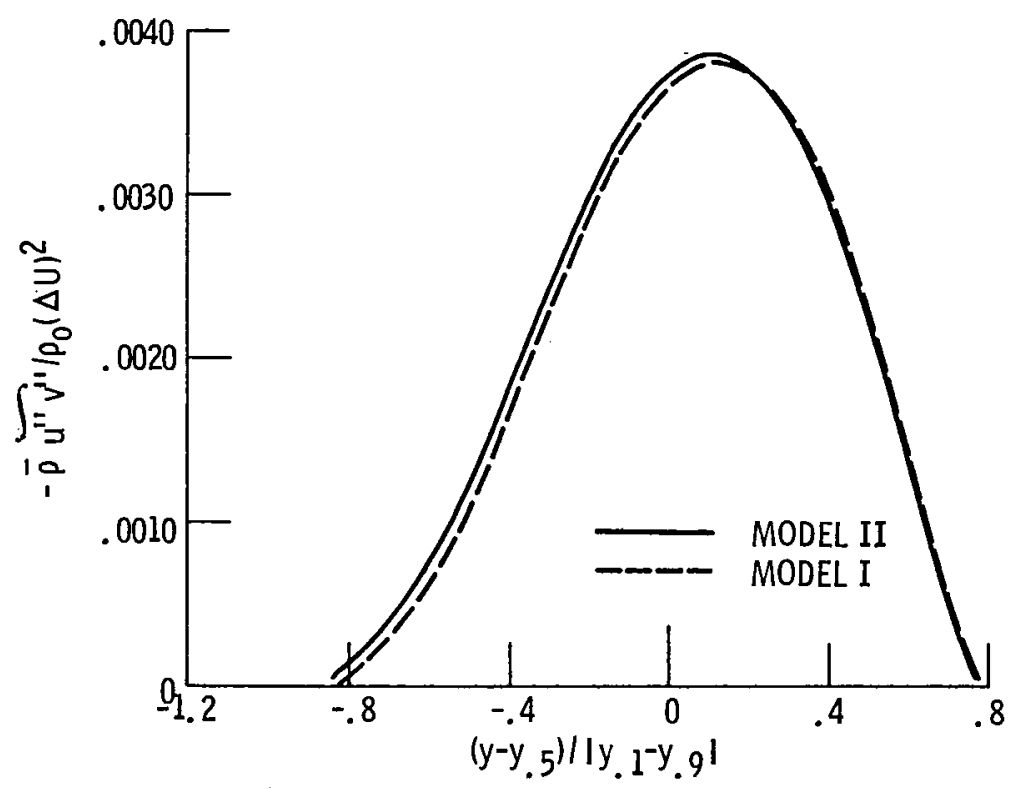

Figure 6. - Comparison of turbulent shear stress prediction of model I and II for high heat release case, $\varphi=1 / 4$. 


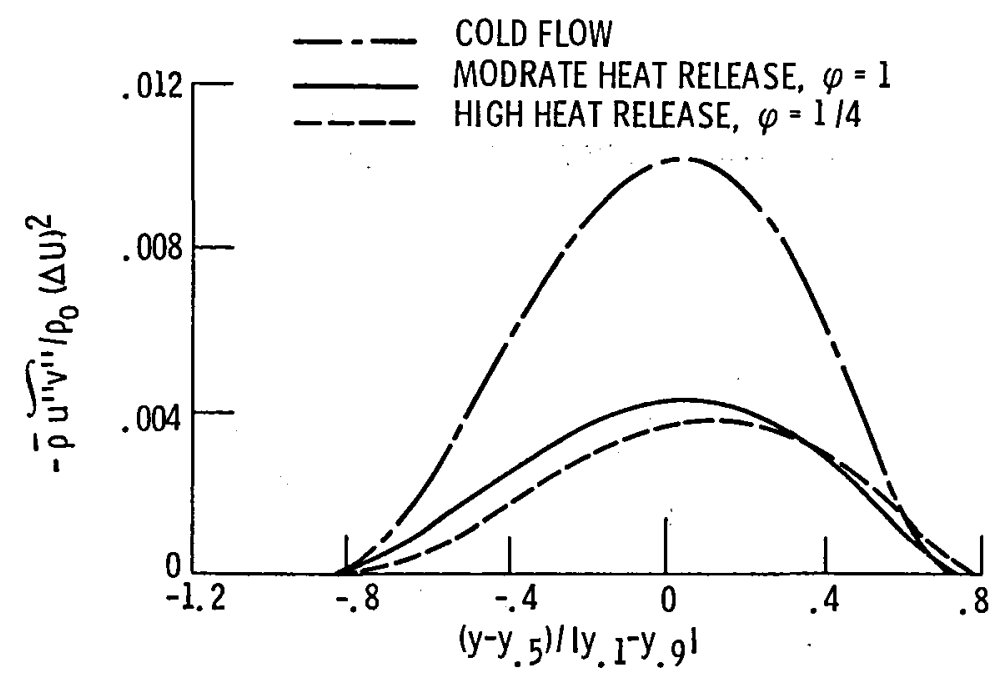

Figure 7. - Comparison of turbulent shear stress for different heat release values, using model I.

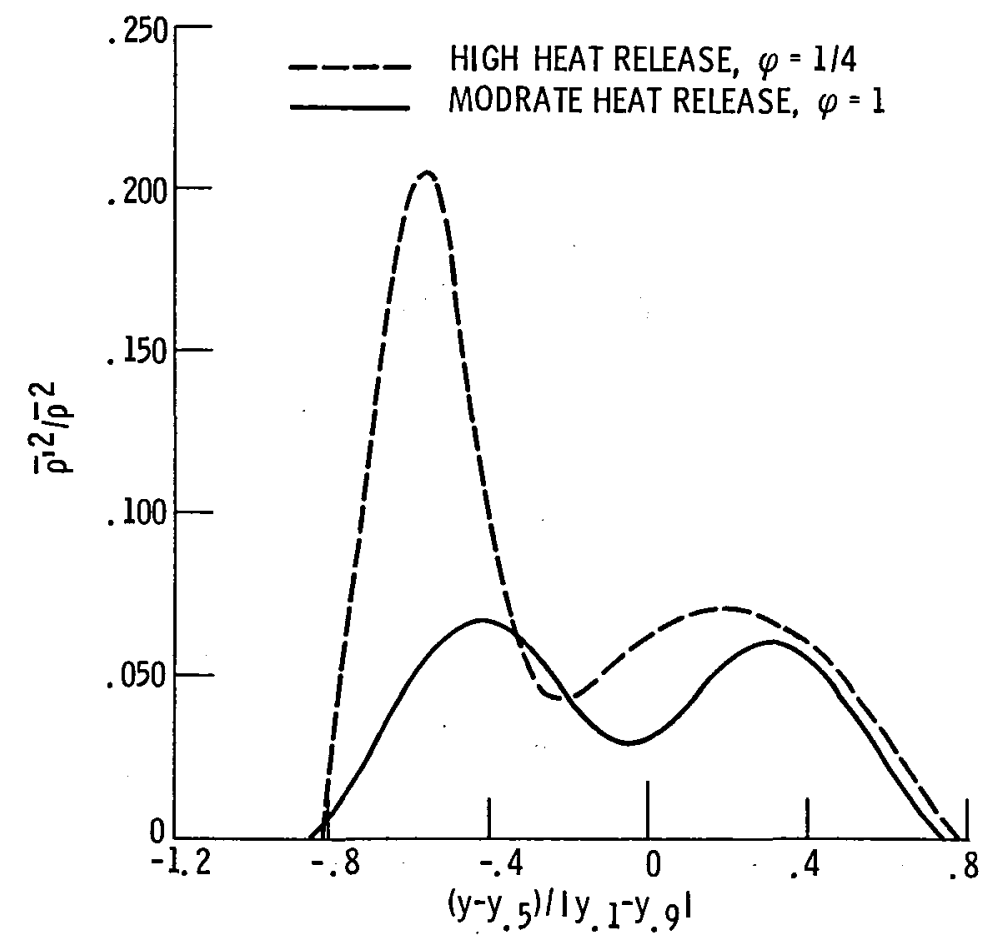

Figure 8. - Comparison of density variances for different equivalence ratios, using model I. 


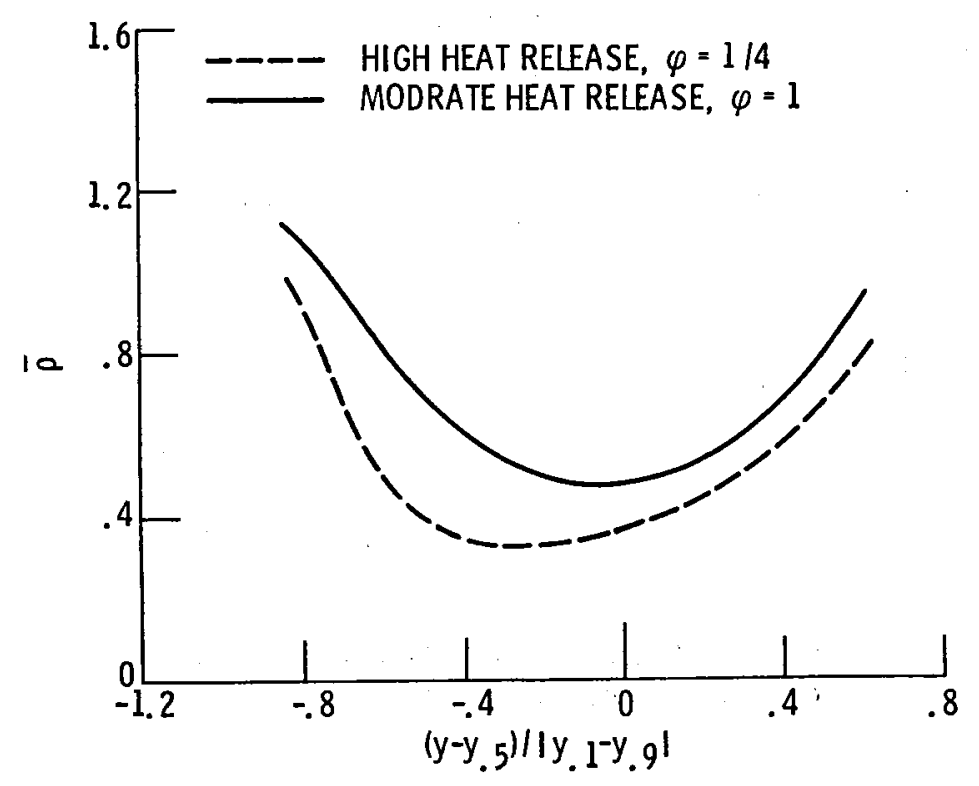

Figure 9. - Comparison of mean densities for different equivalence ratios, using model I.

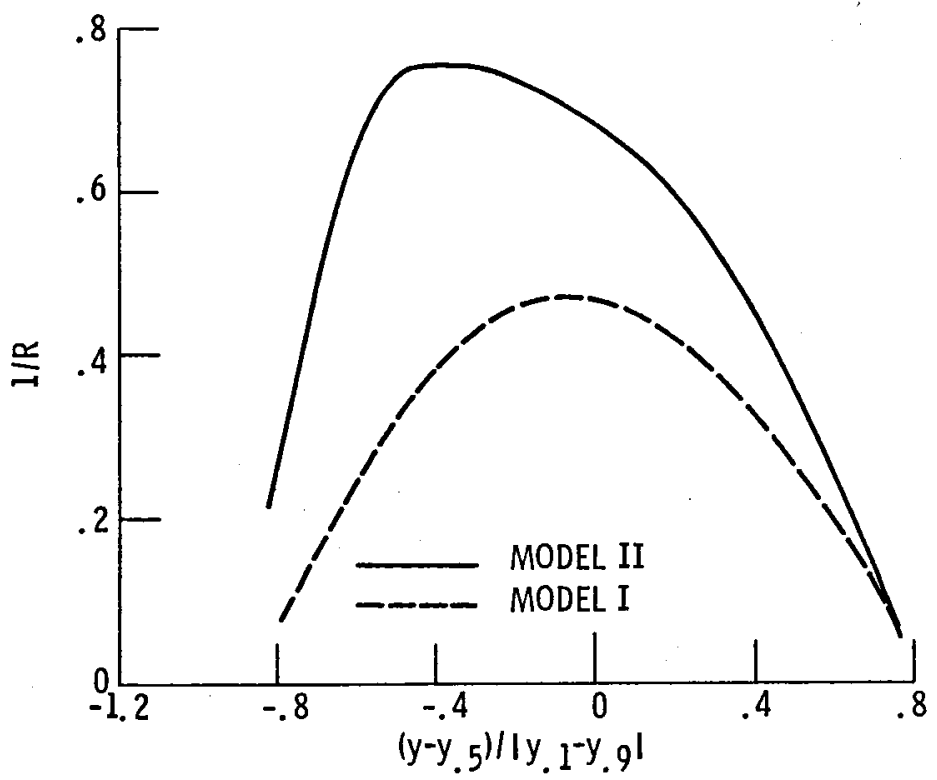

Figure 10. - Ratio of scalar to velocity time scales for high heat release case, $\varphi=1 / 4$. 


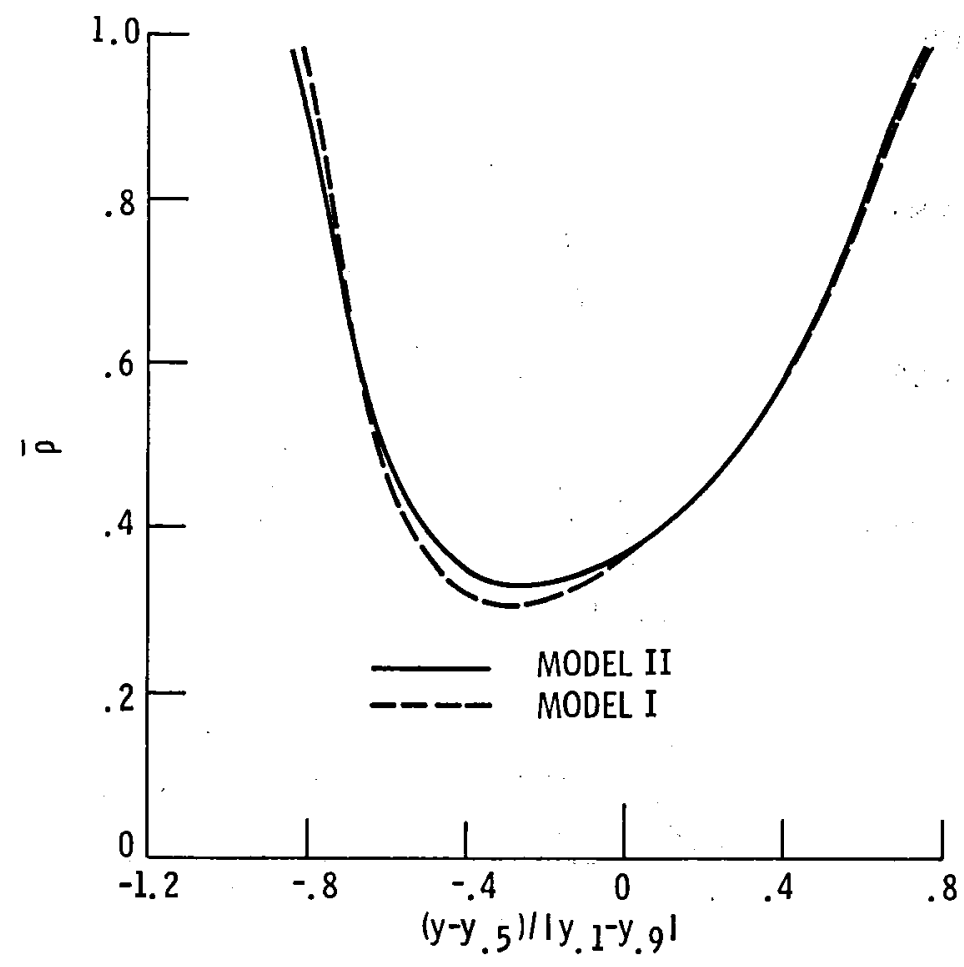

Figure 1l. - Comparison of density predictions for high heat release case, $\varphi=1 / 4$.

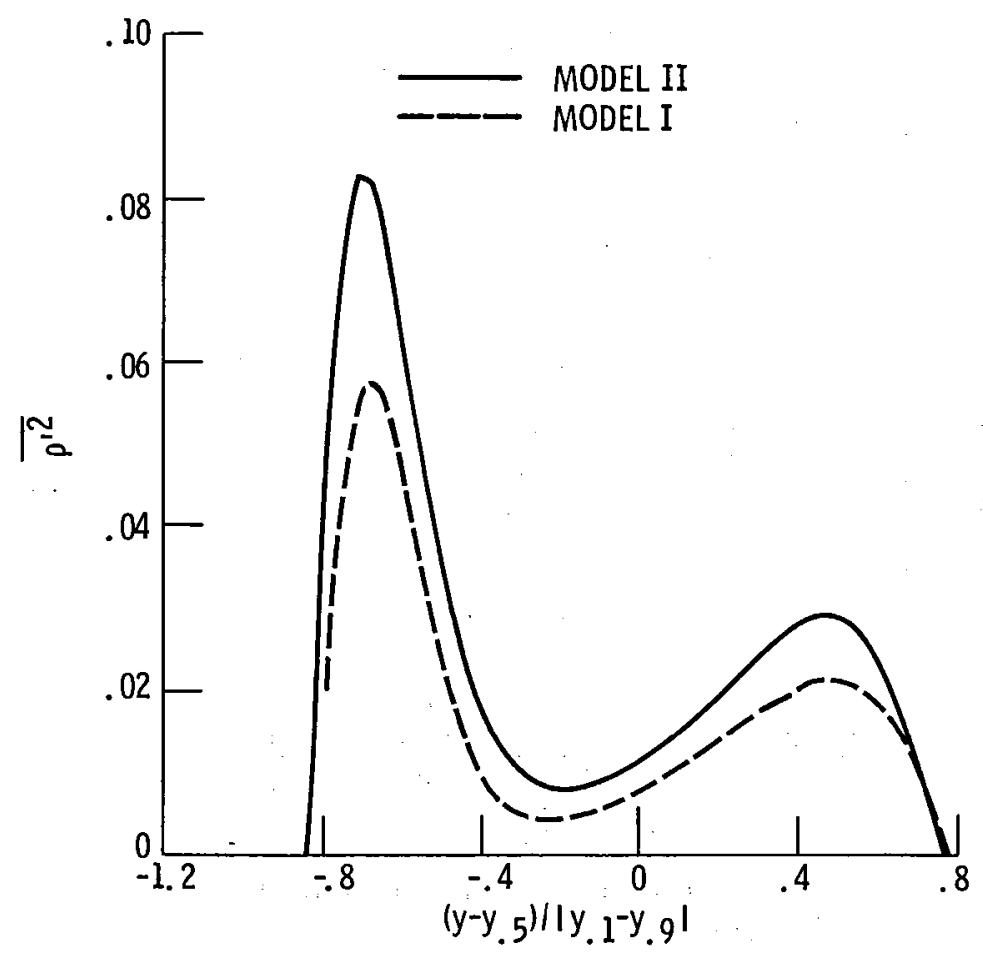

Figure 12. - Comparison of density variance predictions for high heat release case, $\varphi=1 / 4$. 


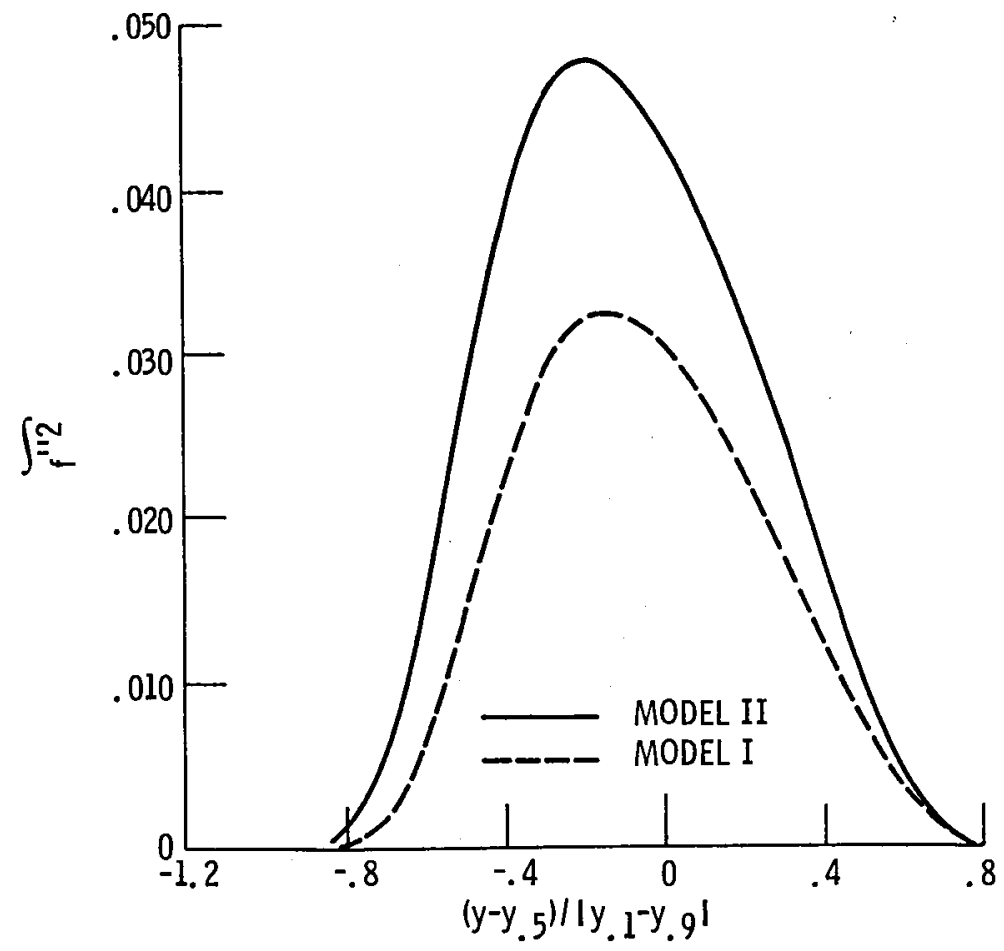

Figure 13. - Mixture fraction variance for high heat release case, $\varphi=1 / 4$.

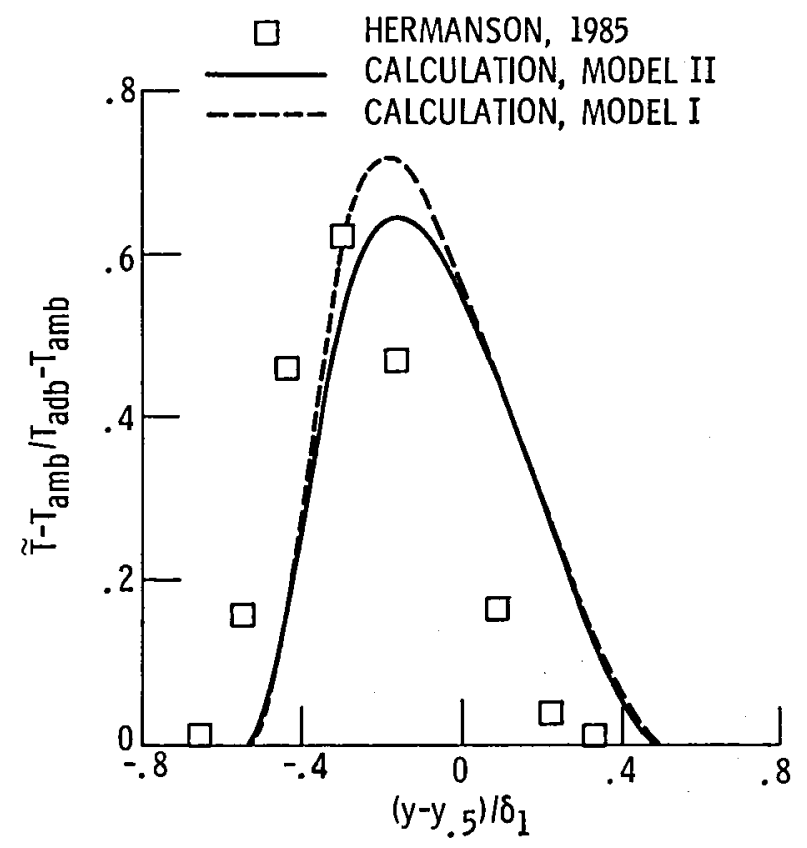

Figure 14. - Temperature comparison for high heat release case, $\varphi=1 / 4$. 


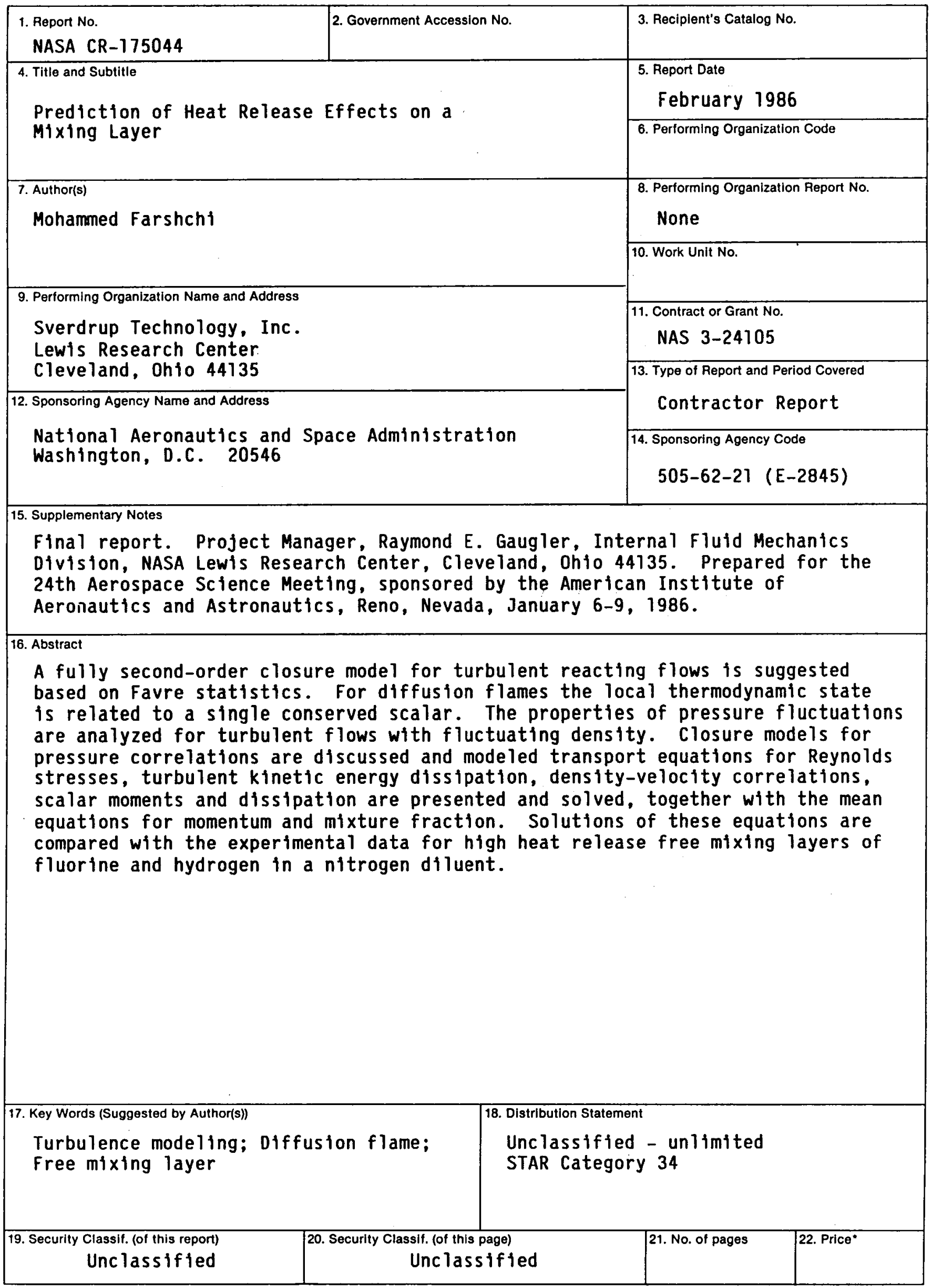

"For sale by the National Technical Information Service, Springfield, Virginia 22161 
End of Document 